\title{
Rapid Response to Pembrolizumab in a Chemo-Refractory Testicular Germ Cell Cancer with Microsatellite Instability-High
}

\author{
Koji Kawai \\ Akinobu Tawada ${ }^{2}$ \\ Mizuki Onozawa' \\ Takamitsu Inoue' \\ Hiromichi Sakurai' \\ Ichiro Mori ${ }^{3}$ \\ Yuichi Takiguchi ${ }^{2}$ \\ Jun Miyazaki'
}

'Department of Urology, International University of Health and Welfare Narita Hospital, Narita, Japan; ${ }^{2}$ Department of Medical Oncology, Graduate School of Medicine, Chiba University, Chiba, Japan; ${ }^{3}$ Department of Pathology, International University of Health and Welfare Narita Hospital, Narita, Japan
Correspondence: Jun Miyazaki

Department of Urology, International University of Health and Welfare Narita Hospital, 852 Hatakeda, Narita, Chiba, 286-0I 24, Japan

Tel $+81-47-635-5600$

Fax +8I-47-635-5586

Email jmiyazaki@iuhw.ac.jp

\begin{abstract}
Testicular germ cell tumor (TGCT) is highly chemo-sensitive cancer; however, there is no established treatment for TGCT relapsed after multiple chemotherapy. Although pembrolizumab showed durable stable disease in some patients, no reliable biomarker for predicting response is available. High microsatellite instability (MSI) is rare in chemo-naïve TGCT. We report a TGCT patient with a rapid response to pembrolizumab. A 34-year-old Japanese male diagnosed with advanced TGCT underwent PCR-based testing of the primary site; it did not reveal MSI. He relapsed after four chemotherapy regimens: bleomycin, etoposide and cisplatin; paclitaxel, ifosfamide and cisplatin; vinblastine, ifosfamide and cisplatin; and irinotecan+nedaplatin with a total of 20 treatment cycles. Chemotherapy was thus discontinued. Re-examination by a CT-guided needle biopsy for progressing retroperitoneal lymph node (RPLN) metastases showed MSI-high; pembrolizumab was initiated. After only two doses, the human chorionic gonadotropin level decreased from 6500 to $<1.0$ IU/L. PET-CT showed shrinkage of the RPLN metastases with diminished metabolism. The patient is currently free from disease progression for 6 months from the start of pembrolizumab. This is the first report of refractory TGCT with MSI-high responding to pembrolizumab. We emphasize the utility of a metastatic-site biopsy to check the MSI status for refractory TGCT even when primary site is MSI-negative.
\end{abstract}

Keywords: pembrolizumab, testicular cancer, microsatellite instability

\section{Introduction}

Testicular germ cell tumor (TGCT) is the most common malignancy in young adult men. TGCT is a highly chemo-sensitive cancer, and up to $80 \%$ of patients with advanced disease can be cured by initial chemotherapy and surgery. ${ }^{1}$ Even if the disease relapses after initial treatment, patients have a chance of cure with second-line chemotherapy; however, there is no established treatment for patients who have further relapse. ${ }^{2,3}$ Given the low efficacy of existing chemotherapeutic and molecularly targeted drugs for chemo-refractory TGCT, investigations into novel therapies are ongoing. One of the promising candidate families is the immune check-point inhibitors (ICIs) targeting programmed cell death protein-1 (PD-1) and programmed cell death ligand-1 (PD-L1) pathways.

There are several case reports and case series on the clinical responses of germ cell tumor (GCT) patients to pembrolizumab or nivolumab, and these reports describe the use of ICIs as a prime target. ${ }^{4-8}$ Of the four contemporary GCT clinical studies using ICIs, ${ }^{9-12}$ two tested the effect of the anti-PD-L1 antibodies 
durvalumab and avelumab. ${ }^{9,10}$ In both studies, ICI monotherapy resulted in no clinical response. The other two studies tested the efficacy of pembrolizumab; no partial or complete responses were observed in 24 refractory GCT patients, ${ }^{11,12}$ but five patients achieved relatively longterm stable disease (range 19 weeks-11 months). ${ }^{11,12}$ While the proportion of patients who benefitted from pembrolizumab was low, the results showed that the drug is certainly worth considering if effective biomarkers for predicting the response are available.

With respect to suitable biomarkers, PD-L1 expression on cancer cells is the logical choice for predicting the response to anti-PD-1 or anti-PD-L1 therapy. ${ }^{13}$ However, results from a Phase II study for pembrolizumab showed that PD-L1 expression may not be a truly reliable predictive biomarker for refractory GCT. ${ }^{11}$ A recent metaanalysis of randomized control studies for various cancer types suggested that PD-L1 expression status alone is an insufficient predictive biomarker. ${ }^{14}$ As other biomarkers, higher tumor mutational burden (TMB) and microsatellite instability (MSI)-high status were associated with favorable clinical outcomes after anti-PD-1/PD-L1 therapy for various types of cancer, ${ }^{15}$ but high TMB is known to be extremely rare in GCT. MSI-high is also rare, ${ }^{16,17}$ but several studies suggested the incidence is higher in chemorefractory TGCT compared to the chemo-naïve primary tumor. $^{18,19}$

Herein, we describe a TGCT patient with histologically proven MSI-high status who had a rapid response to pembrolizumab. To our knowledge, this is the first report of refractory GCT with MSI-high status responding to pembrolizumab. We also discuss a rationale of cell-free DNA (cfDNA) next-generation sequencing (NGS) for detecting the MSI-high status in this clinical setting. ${ }^{20,21}$

\section{Case Report}

A 34-year-old Japanese male with advanced testicular cancer was referred to Chiba University Hospital in December 2017 for chemotherapy. Computed tomography (CT) revealed multiple lung metastases and retroperitoneal lymph node (RPLN) metastases. Laboratory testing showed a human chorionic gonadotropin (hCG) level of 39,400 IU/L, lactate dehydrogenase (LDH) $528 \mathrm{IU} / \mathrm{L}$, and alpha-fetoprotein (AFP) $3.9 \mathrm{ng} / \mathrm{mL}$. The pathological diagnosis of the primary site was pure seminoma, and polymerase chain reaction (PCR)-based MSI testing using five microsatellite markers (MONO-27, BAT25, BAT26, NR21, and NR-24) did not reveal MSI.
The patient received multiple chemotherapy using four different regimens with a total of 20 treatment cycles: bleomycin, etoposide and cisplatin, paclitaxel, ifosfamide and cisplatin, vinblastine, ifosfamide and cisplatin, and a combination of irinotecan+nedaplatin. The disease nevertheless progressed after the last chemotherapy in December 2019, and the patient's hCG level increased to 19,764 IU/L. Because of the definitive chemo-refractory disease, the chemotherapy was discontinued.

At this point, the patient's MSI status was re-checked by a commercially available cfDNA NGS assay, ${ }^{20,21}$ the Guardant360 (Guardant Health, Redwood City, CA), which resulted in positive MSI-high status. As other molecular findings of interest, Guardant360 revealed an insertion mutation and a nonsense mutation in AT-rich interactive domain-containing protein 1A (ARID1A) gene, also it showed an insertion mutation BRCA2 gene. Those gene alterations are potential marker for the efficacy of Poly (ADP-ribose) polymerase (PARP) inhibitors, but RARP inhibitors are not available for TGCT in clinical setting. Therefore, treatment with pembrolizumab was considered, but unexpectedly, the patient's metastases regressed without any additional treatment. As shown in Figure 1, between January 2020 and June 2020, CT images revealed that both lung and RPLN metastases spontaneously regressed, and the hCG level decreased from 84,920 to $1402 \mathrm{IU} / \mathrm{L}$. However, the level had increased to $2407 \mathrm{IU} / \mathrm{L}$ at 2 months later.

The patient was then referred to our hospital in September 2020 for further management. As shown in Figure 2, his hCG level continuously increased. Positron emission tomography (PET)-CT revealed the reenlargement of RPLN metastases with hypermetabolism, but the lung metastases had completely disappeared. Due to the positive result on the Guardant360 assay, we decided to re-examine the patient's MSI status by performing a CT-guided needle biopsy of the progressing RPLN metastases.

As shown in Figure 3, the histological examination of the biopsy specimens revealed mixed germ cell cancer, and the VENTANA (SP-142) immunohistochemistry assay (Roche Diagnostics, Indianapolis, IN) result was positive for PD-L1 expression. In addition, MSI testing, ${ }^{22}$ using a quasi-monomorphic variation range showed MSI in four (MONO-27, BAT25, BAT26 and NR-21) of five microsatellite markers. The tumors were thus classified as MSI-high. Immunotherapy with pembrolizumab was then initiated, and as shown in Figure 2, the 


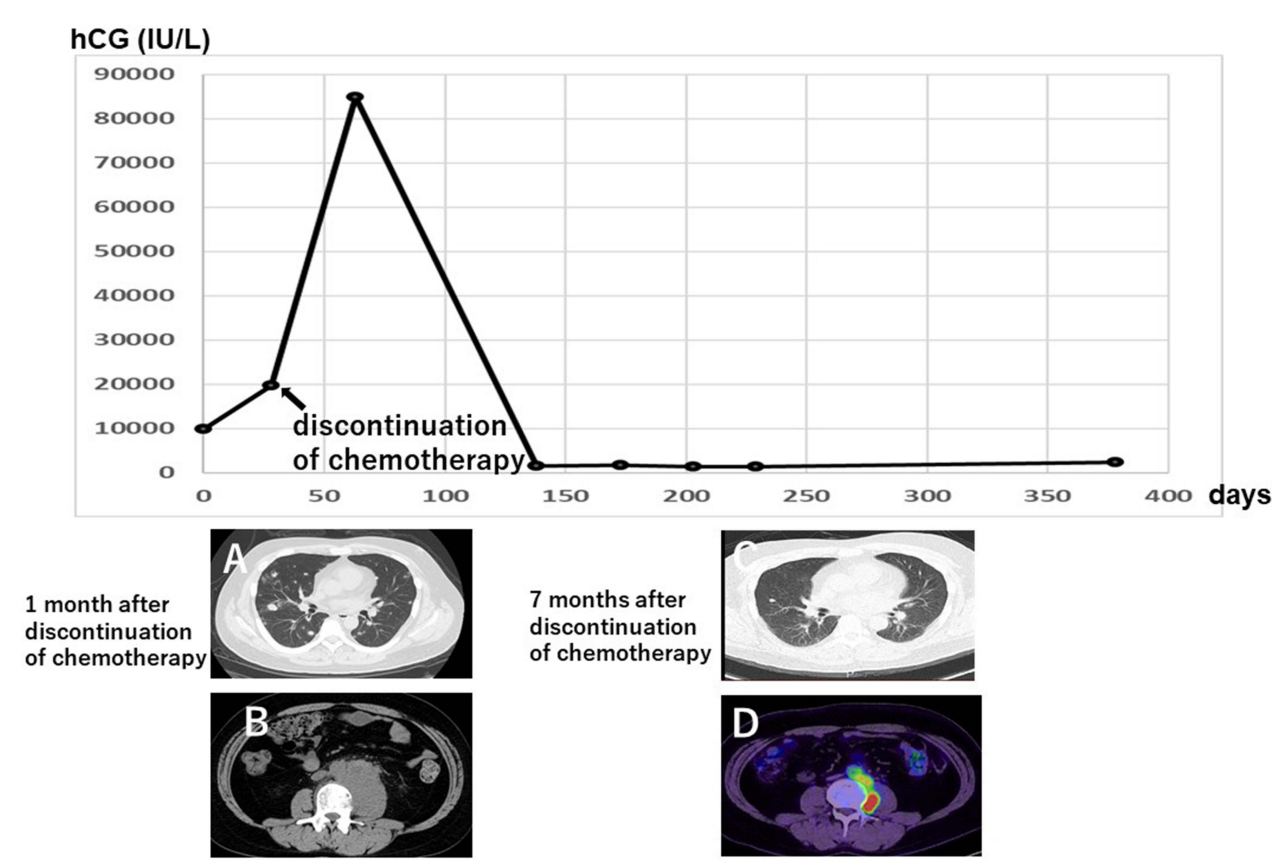

Figure I Spontaneous regression of metastases after the discontinuation of chemotherapy. Plain CT scans in cross-sectional views of lung metastases and retroperitoneal lymph node (RPLN) metastases at I month after chemotherapy discontinuation (A and B). Plain CT of lung metastases (C) and PET-CT of RPLN metastases (D) at 7 months after chemotherapy discontinuation. Both lung and RPLN metastases spontaneously regressed without further treatment. During this time, the patient's hCG level decreased from 84,920 to $1402 \mathrm{IU} / \mathrm{L}$.

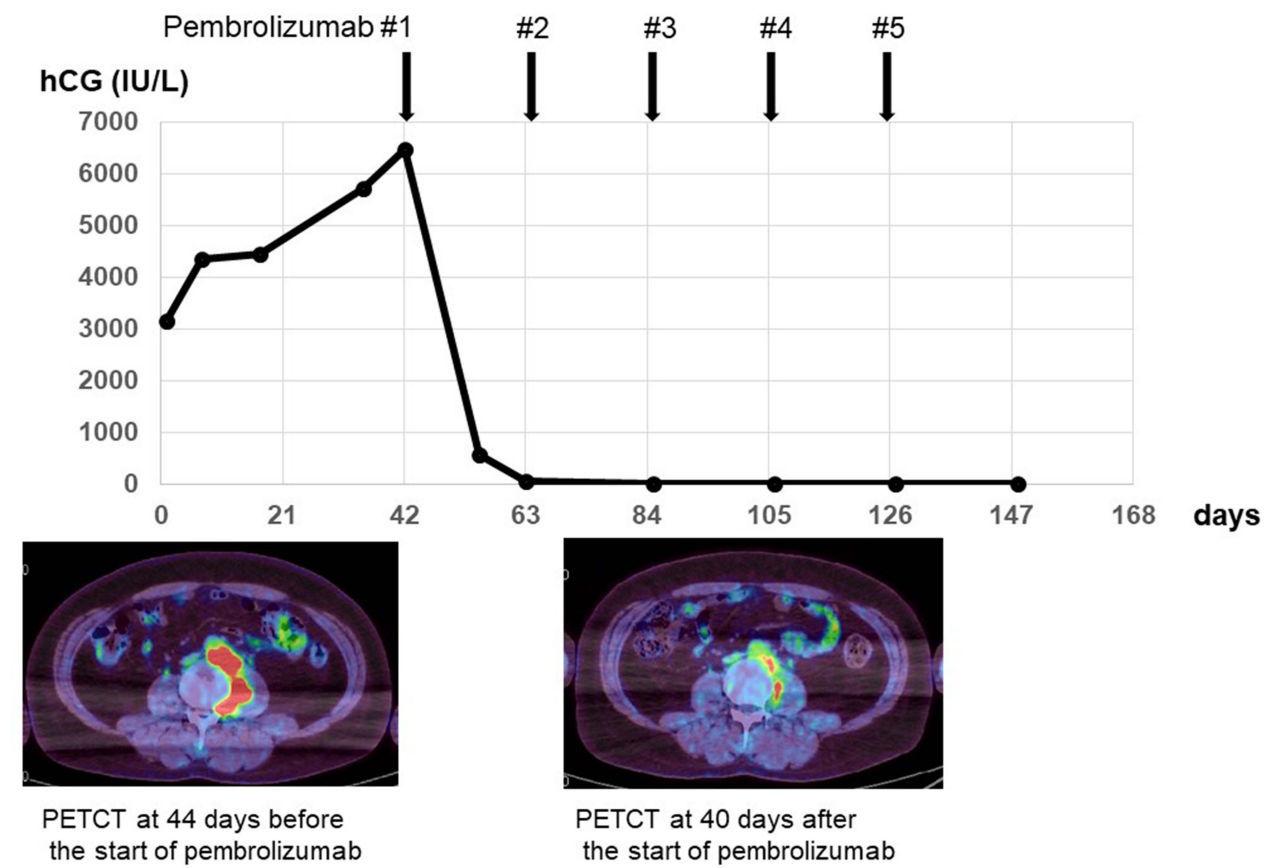

Figure 2 Clinical course of treatment with pembrolizumab. Since the patient's hCG level started to re-increase after spontaneous regression continued for 8 months, immunotherapy with pembrolizumab was started. The hCG level decreased from 6500 to $<1.0 \mathrm{IU} / \mathrm{L}$ after two doses of pembrolizumab. PET-CT 40 days after the start of treatment showed shrinkage of RPLN metastases with diminished metabolism.

hCG level decreased from $6500 \mathrm{IU} / \mathrm{L}$ to $<1.0 \mathrm{IU} / \mathrm{L}$ after only two doses of pembrolizumab. PET-CT showed the shrinkage of RPLN metastases with diminished metabolism. Since asymptomatic organizing pneumonia developed, pembrolizumab was discontinued after 6 doses administration. The patient is currently free from 


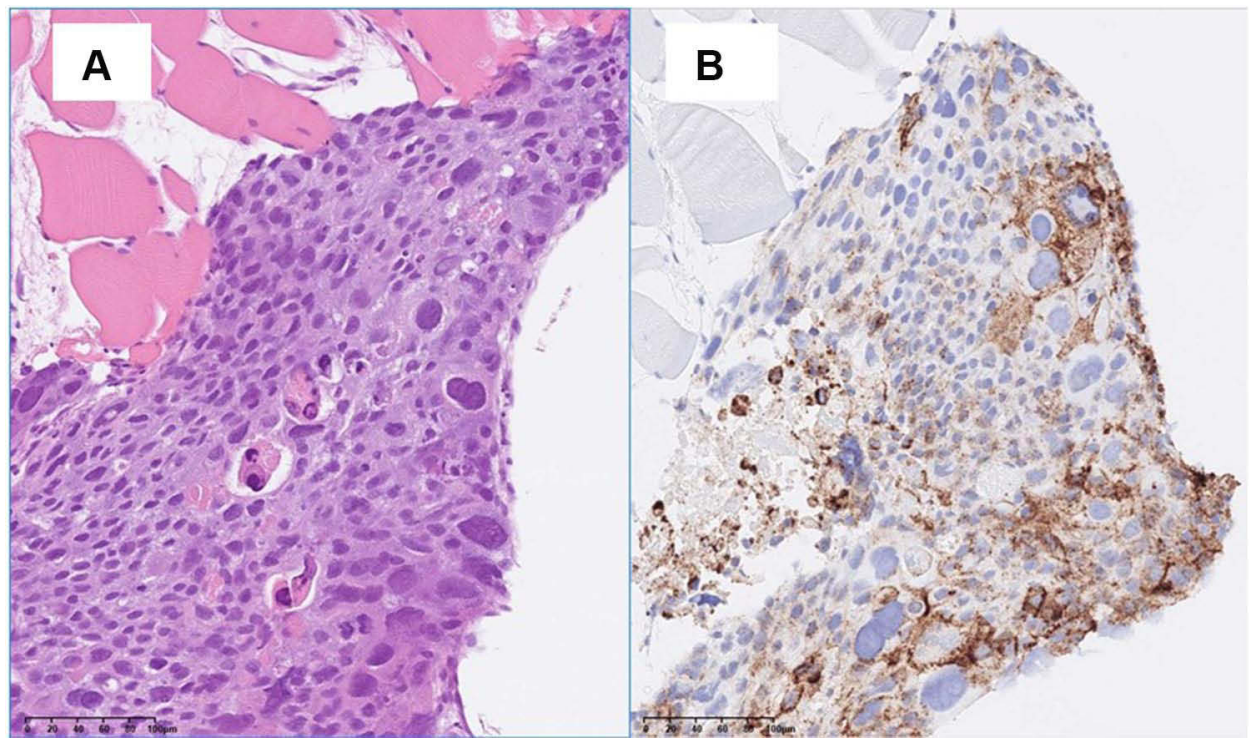

Figure 3 Pathological section of RPLN metastasis biopsy. The biopsy specimen showed the infiltration of mixed germ cell cancer. Immunohistochemistry revealed strong positive staining for PD-LI. (A) Hematoxylin and eosin staining. (B) VENTANA (SP-I42) immunohistochemistry assay, magnification: 200x.

disease progression for 6 months from the start of pembrolizumab.

\section{Discussion}

In the present case, a CT-guided needle biopsy for RPLN metastases demonstrated the MSI-high status, suggesting that pembrolizumab might yield clinical benefit. The patient had a rapid response, as his hCG level decreased from 6500 to $<1.0 \mathrm{IU} / \mathrm{L}$ after only two doses of pembrolizumab. Since the preceding histological examination of the patient's primary tumor did not show MSI, the decision to re-examine the patient's MSI status by needle biopsy for progressing metastatic site was not easy, as this procedure is not free from potential complications. The positive data obtained by the Guardant360 assay was an important clue for making the decision to re-examine the MSI status.

Pembrolizumab has provided improved patient responses in various types of cancer that have a high level of PD-L1 expression. ${ }^{13}$ In TGCT, two studies reported higher levels of PD-L1 expression compared to normal testes, ${ }^{23,24}$ and both studies showed that high PDL1 expression was associated with unfavorable survival. $^{23,24}$ To date, five patients with refractory GCT were reported to partially respond to anti-PD-1 therapy. ${ }^{4-8}$ Of them, four showed positive PD-1 expression. These findings suggested that PD-1 expression is an effective biomarker for refractory GCT, but Adra et al reported that two of 12 patients treated with pembrolizumab achieved radiographic stable disease, and both had negative PD-L1 staining. ${ }^{11}$ Two patients had positive PDL1 expression, and progressive disease was their best response. A meta-analysis of randomized control studies for various cancer types also suggested that the PDL1 expression status alone is insufficient as a predictive biomarker. $^{14}$ PD-L1 expression may thus not be a reliable predictive biomarker for refractory GCT.

As other biomarkers, the higher TMB and MSI-high status are effective predictors for the response to anti-PD -1/PD-L1 therapy. The reported proportion of positive cases for both markers in TGCT are extremely low; however, several studies suggested that the MSI-high incidence is higher in chemo-refractory tumors compared to chemo-naïve primary tumors. Carcano et al reported that MSI was completely absent in 133 primary tumors examined before chemotherapy, ${ }^{16}$ whereas Honecker et al showed MSI-high in $26 \%$ of 35 chemo-resistant tumors of GCT patients; most had been treated by first-line or salvage high-dose chemotherapy. ${ }^{18}$ MSI-high status was observed in four of eight late relapsed patients after chemotherapy, ${ }^{19}$ but Necchi et al reported only one $(2 \%)$ MSI-high case among 51 chemotherapy-treated patients. ${ }^{17}$ Several factors may be involved in this discrepancy, such as differences in technology or the degree of chemoresistance. Histology of MSI-high tumors is another discussion point.

The reported incidences of MSI-high do not support a routine biopsy for progressing chemo-resistant tumors, because it is not free from potential complications. As in 
the present case, the Guardant360cfDNA NGS assay is useful for identifying a biopsy candidate to examine the MSI status in clinical practice. MSI detection by the Guardant360 is reported to be highly concordant with tissue-based testing. ${ }^{20,21}$ The FDA approved the Guardant360 as a diagnostic tool to guide treatment options for non-small-cell lung cancer.

Our patient had experienced a dramatic spontaneous regression of lung metastases before the progression of RPLN metastases. In TGCT, a spontaneous regression of metastatic sites is extremely rare, ${ }^{25,26}$ but spontaneous regression of the primary tumor, so-called burned out tumor, is a well-described phenomenon. ${ }^{27}$ Lymphoplasmacytic infiltrates in the scars are commonly observed in regressed tumors. In the present case, pathology of testicular tumor was pure seminoma, but biopsy of RPLN metastases revealed mixed germ cell cancer. Since pretreatment hCG level was extraordinary high considering pure seminoma, we suspected presence of non-seminoma elements in metastatic sites. This is probably result of differentiation from seminoma to nonseminoma in metastatic sites. As another explanation, there is a possibility that non-seminoma elements in primary site had spontaneously regressed. Although the mechanism underlying spontaneous regression is unclear, these findings may indicate that some TGCT cases have immunogenic activity that facilitates the response to anti-PD-1/PD-L1 therapy.

\section{Conclusion}

In summary, our patient's case suggests that pembrolizumab is effective for refractory TGCT with MSI-high status, as other MSI-high cancers may be. We emphasize that a biopsy of metastatic sites to check the MSI status is one of the choices for refractory TGCT even when the primary site is negative for MSI. The cfDNA assay is an important clue for deciding whether to perform a biopsy for metastatic sites. Although further studies to examine concordance of MSI detection by the cfDNA with tissue-based testing are needed, the present case suggests that if MSI-H is identified with cfDNA that may be also reasonable rationale to proceed with immunotherapy for TGCT.

\section{Abbreviations}

TGCT, Testicular germ cell tumor; MSI, microsatellite instability; RPLN, retroperitoneal lymph node; ICIs, immune check-point inhibitors; PD-L1, programmed cell death ligand-1; cfDNA, cell-free DNA; NGS, nextgeneration sequencing; hCG, human chorionic gonadotropin; AFP, alpha-fetoprotein; LDH, lactate dehydrogenase.

\section{Ethical Approval}

Institutional approval was not required to publish the case details.

\section{Patient Consent for Publication}

Written informed consent was obtained from the patient.

\section{Acknowledgments}

The authors thank the patient for his permission to publish this case report.

\section{Disclosure}

Dr Yuichi Takiguchi reports grants, personal fees from Chugai pharmaceutical Co, MSD, Taiho pharmaceutical Co, AstraZeneca, and Ono Pharmaceutical Co., during the conduct of the study; grants, personal fees from Eli Lilly, Takeda, Daiichi Sankyo, Novartis, Kyowa-Hakko Kirin, and Boehringer Ingelheim, outside the submitted work. The authors have no other conflicts of interest to disclose.

\section{References}

1. Hanna NH, Einhorn LH. Testicular cancer-discoveries and updates. $N$ Engl J Med. 2014;371:2005-2016. doi:10.1056/NEJMra1407550

2. Feldman DR, Patil S, Trinos MJ, et al. Progression-free and overall survival in patients with relapsed/refractory germ cell tumors treated with single-agent chemotherapy: endpoints for clinical trial design. Cancer. 2012;118:981-986. doi:10.1002/cncr.26375

3. Oing C, Alsdorf WH, von Amsberg G, et al. Platinum-refractory germ cell tumors: an update on current treatment options and developments. World J Urol. 2017;35:1167-1175. doi:10.1007/s00345-016-1898-z

4. Shah S, Ward JE, Bao R, et al. Clinical response of a patient to anti-PD-1 immunotherapy and the immune landscape of testicular germ cell tumors. Cancer Immunol Res. 2016;4:903-909. doi:10.1158/2326-6066.CIR-16-0087

5. Zschäbitz S, Lasitschka F, Jäger D, et al. Activity of immune checkpoint inhibition in platinum refractory germ-cell tumors. Ann Oncol. 2016;27:1356-1360. doi:10.1093/annonc/mdw146

6. Chi EA, Schweizer MT. Durable response to immune checkpoint blockade in a platinum-refractory patient with nonseminomatous germ cell tumor. Clin Genitourin Cancer. 2017;15:e855-e857. doi:10.1016/j.clgc.2017.04.005

7. Aksun MS, Ucgul E, Sahin TK, et al. Nivolumab for the salvage treatment of desperate germ cell tumor: a case report. J Oncol Pharm Pract. 2020;6:1078155220977638. doi:10.1177/1078155220977638

8. Zschäbitz S, Lasitschka F, Hadaschik B, et al. Response to anti-programmed cell death protein-1 antibodies in men treated for platinum refractory germ cell cancer relapsed after high-dose chemotherapy and stem cell transplantation. Eur J Cancer. 2017;76:1-7. doi:10.1016/j.ejca.2017.01.033

9. Necchi A, Giannatempo P, Raggi D, et al. An open-label randomized Phase 2 study of durvalumab alone or in combination with tremelimumab in patients with advanced germ cell tumors (APACHE): results from the first planned interim analysis. Eur Urol. 2019;75:201-203. doi:10.1016/j.eururo.2018.09.010

10. Mego M, Svetlovska D, Chovanec M, et al. Phase II study of avelumab in multiple relapsed/refractory germ cell cancer. Invest New Drugs. 2019;37:748-754. doi:10.1007/s10637-019-00805-4 
11. Adra N, Einhorn LH, Althouse SK, et al. Phase II trial of pembrolizumab in patients with platinum refractory germ-cell tumors: a Hoosier Cancer Research Network Study GU14-206. Ann Oncol. 2018;29:209-214. doi:10.1093/annonc/mdx680

12. Tsimberidou AM, Vo HH, Subbiah V, et al. Pembrolizumab in patients with advanced metastatic germ cell tumors. Oncologist. 2021;26(7):558. doi:10.1002/onco.13682

13. Carbognin L, Pilotto S, Milella M, et al. Differential activity of nivolumab, pembrolizumab and MPDL3280A according to the tumor expression of programmed death-ligand-1 (PD-L1): sensitivity analysis of trials in melanoma, lung and genitourinary cancers. PLoS One. 2015;10(6):e0130142. doi:10.1371/journal.pone.0130142

14. Shen X, Zhao B. Efficacy of PD-1 or PD-L1 inhibitors and PD-L1 expression status in cancer: meta-analysis. BMJ. 2018;362:k3529. doi:10.1136/bmj.k3529

15. Yarchoan M, Hopkins A, Jaffee EM. Tumor mutational burden and response rate to PD-1 inhibition. $N$ Engl $J$ Med. 2017;377: 2500-2501. doi:10.1056/NEJMc1713444

16. Cárcano FM, Lengert AH, Vidal DO, et al. Absence of microsatellite instability and BRAF (V600E) mutation in testicular germ cell tumors. Andrology. 2016;4:866. doi:10.1111/andr.12200

17. Necchi A, Bratslavsky G, Corona RJ, et al. Genomic characterization of testicular germ cell tumors relapsing after chemotherapy. Eur Urol Focus. 2020;6:122-130. doi:10.1016/j.euf.2018.07.013

18. Honecker F, Wermann H, Mayer F, et al. Microsatellite instability, mismatch repair deficiency, and BRAF mutation in treatment-resistant germ cell tumors. J Clin Oncol. 2009;27:2129-2136. doi:10.1200/ JCO.2008.18.8623

19. Mayer F, Wermann H, Albers P, et al. Histopathological and molecular features of late relapses in non-seminomas. BJU Int. 2011;107:936-943. doi:10.1111/j.1464-410X.2010.09631.x
20. Leighl NB, Page RD, Raymond VM, et al. Clinical utility of comprehensive cell-free DNA analysis to identify genomic biomarkers in patients with newly diagnosed metastatic non-small cell lung cancer. Clin Cancer Res. 2019;25::4691-4700. doi:10.1158/1078-0432.CCR19-0624

21. Willis J, Lefterova M, Artyomenko A, et al. Validation of microsatellite instability detection using a comprehensive plasma-based genotyping panel. Clin Cancer Res. 2019;25:7035-7045. doi:10.1158/ 1078-0432.CCR-19-1324

22. Bando H, Okamoto W, Fukui $T$, et al. Utility of the quasi-monomorphic variation range in unresectable metastatic colorectal cancer patients. Cancer Sci. 2018;109:3411-3415. doi:10.1111/ cas. 13774

23. Fankhauser CD, Curioni-Fontecedro A, Allmann V, et al. Frequent PD-L1 expression in testicular germ cell tumors. $\mathrm{Br} J$ Cancer. 2015;113:411-413. doi:10.1038/bjc.2015.244

24. Cierna Z, Mego M, Miskovska V, et al. Prognostic value of programmed-death-1 receptor (PD-1) and its ligand 1 (PD-L1) in testicular germ cell tumors. Ann Oncol. 2016;27:300-305. doi:10. 1093/annonc/mdv574

25. Birkhead BM, Scott RM. Spontaneous regression of metastatic testicular cancer. Cancer. 1973;32:125-129. doi:10.1002/1097-0142(197307)32:1<125::AID-CNCR2820320117>3.0.CO;2-Y

26. Musser JE, Przybycin CG, Russo P. Regression of metastatic seminoma in a patient referred for carcinoma of unknown primary origin. Nat Rev Urol. 2010;7:466-470. doi:10.1038/nrurol.2010.99

27. Balzer BL, Ulbright TM. Spontaneous regression of testicular germ cell tumors: an analysis of 42 cases. Am J Surg Pathol. 2006;30:858-865. doi:10.1097/01.pas.0000209831.24230.56

\section{Publish your work in this journal}

OncoTargets and Therapy is an international, peer-reviewed, open access journal focusing on the pathological basis of all cancers, potential targets for therapy and treatment protocols employed to improve the management of cancer patients. The journal also focuses on the impact of management programs and new therapeutic agents and protocols on patient perspectives such as quality of life, adherence and satisfaction. The manuscript management system is completely online and includes a very quick and fair peer-review system, which is all easy to use. Visit http://www.dovepress.com/ testimonials.php to read real quotes from published authors. 